\title{
Metastable structures, interplay of sequential deformations, and interactions between tungsten disulfide nanotubes and poly(L-lactide) studied by in situ X-ray scattering
}

\author{
J. A. Kornfield ${ }^{1}$, L. Rocher², A. B. Lennon², A. S. Ylitalo1, T. Di Luccio¹, G. H. Menary², R. Miscioscia ${ }^{3}$, \\ G. De Filippo ${ }^{3}$, G. Pandolfi ${ }^{3}$, F. Villani ${ }^{3}$
}

${ }^{1}$ California Institute of Technology 91125 USA; ${ }^{2}$ Queen's University Belfast, BT9 5AH, UK; ${ }^{3}$ ENEA-Portici, 80055 Italy

kornfield@caltech.edu

Polymer crystallization, particularly near the glass transition, exhibits strong nonlinearities and prolonged metastability that enable fabrication of devices with complex hierarchal structure from $\mathrm{nm}$ to $\mathrm{mm}$. A fascinating example arises in the production of bioresorbable scaffolds (BRS) from poly(L-lactide) (PLLA), in which a sequence of processes (extrusion, stretch-blow molding and crimping) create diverse semicrystalline morphologies, side-by-side within a span of a hundred microns (Figure 1). To discover how these structures form, we need to examine transient structure under conditions that mimic manufacturing processes. An apparatus that enables scattering measurements during the stretch-blow molding step, called "tube expansion" imposes a nearly constant-width elongation as it converts an extruded "preform" into an "expanded tube". To increase the range of accessible properties of PLLA-based BRS, we use this apparatus to examine inorganic nanotubes as potential reinforcing agents that also enhance radiopacity, relevant to clinical applications. Understanding how their microstructure develops during processing is relevant to increasing strength to enable thinner devices and improving radiopacity to enable imaging during implantation. Consistent with the premise of this MS, in-situ X-ray scattering reveals unanticipated phenomena in the transient microstructure of PLLA/WS ${ }_{2} \mathrm{NTs}$ nanocomposites during "tube expansion" (Figure 2).

Surprisingly, the $\mathrm{WS}_{2} \mathrm{NT}$ orientation hardly changes from that produced during extrusion of the preform ( $z$-dir., defined Fig. 1A), despite significant strain in the transverse direction (at inner diameter, $500 \%$ strain in $\theta$ dir.). Although $\mathrm{WS}_{2} \mathrm{NTs}$ promote PLLA nucleation, the NTs do not modify the orientation of crystallization (c-axis along $\theta$, just as observed in tube expansion of neat PLLA). The striking independence of the orientations of the NT and polymer crystals stems may arise from the favorable interaction between PLLA and $\mathrm{WS}_{2} \mathrm{NTs}$ : facile and stable dispersion of $\mathrm{WS}_{2} \mathrm{NTs}$ in PLLA enables strong NT orientation in shear (extrusion); NT that are orthogonal to the stretching direction do not reorient; remaining orthogonal to decouples $\mathrm{WS}_{2} \mathrm{NT}$ orientation from that of PLLA crystals. Future directions include evaluating crossreinforcement of the mutually orthogonal NT and PLLA crystals. Based on the surprising effects we have found, further discoveries likely lie ahead in the effects of $\mathrm{WS}_{2} \mathrm{NT}$ on morphology development during crimping.

\section{Keywords: stretch-blow molding; $\mathrm{WS}_{2} \mathrm{NT}$; flow-induced crystallization; microdiffraction; SAXS/WAXS}

Alla Zak provided $W S_{2} N T$ and guidance. EU Horizon 2020 MC-RISE No 813869 \& 691238. (A.Y.) US NSF GRFP DGE-1745301. (A. Z.) ISF 330-16. 5-ID-D DND-CAT APS.

Acta Cryst. (2021), A77, C575

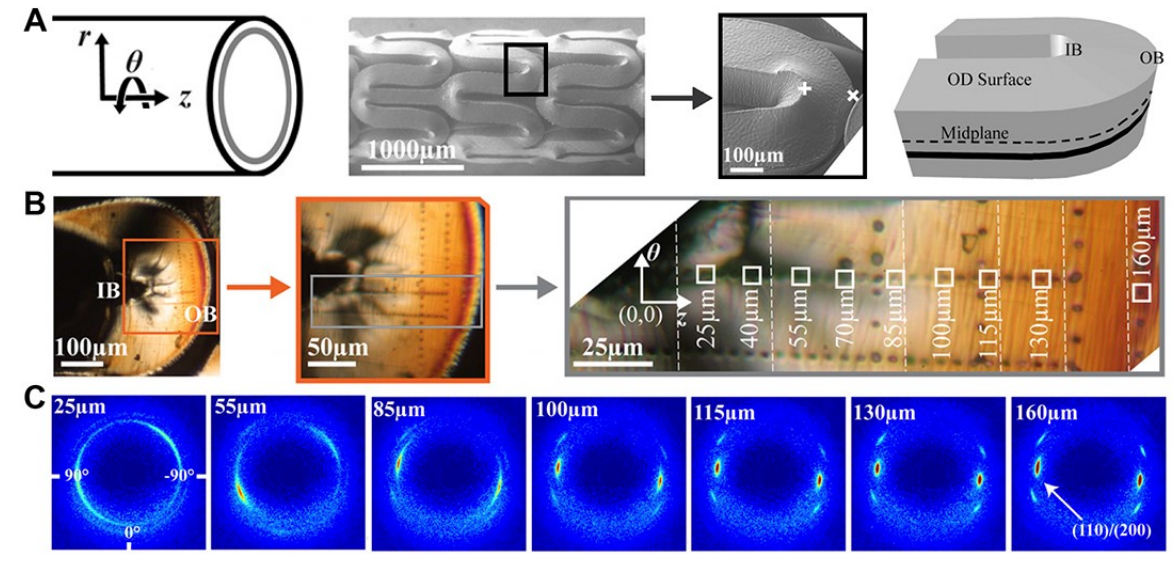

Figure 1. Diverse morphologies form during manufacture of PLLA bioresorbable scaffolds: A. a thin-walled $(150 \mu \mathrm{m})$ tube, created by "tube expansion", is laser cut and crimped. B. Polarized light micrographs guide microdiffraction. C. Morphology changes from weakly-oriented to highly-oriented over $150 \mu \mathrm{m}$.

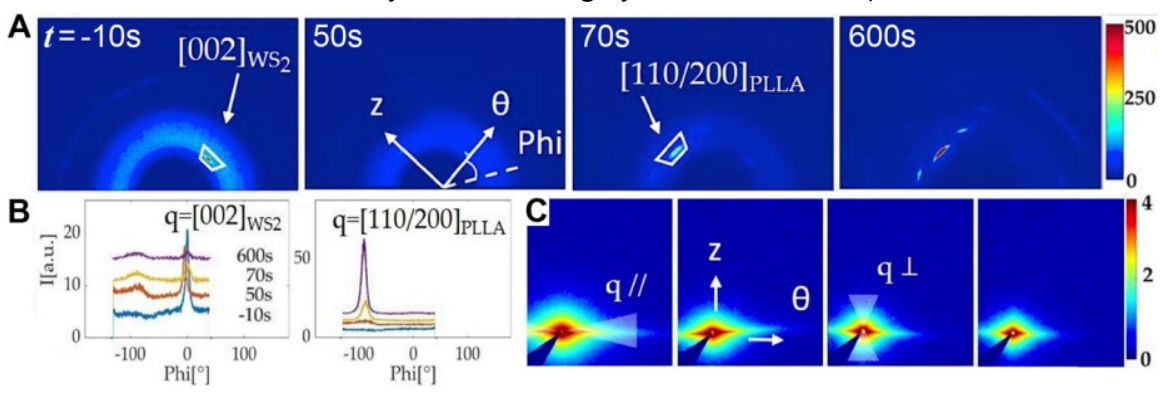

Figure 2. During "tube expansion", $\mathrm{WS}_{2} \mathrm{NT}$ orientation and PLLA crystallization are probed by A,B. WAXS and C. SAXS. Initially, PLLA is amorphous and $\mathrm{WS}_{2} \mathrm{NT}$ are aligned along $z$. Heating begins at $t=0$ from $25^{\circ} \mathrm{C}$ to $80^{\circ} \mathrm{C}$ at $0.5^{\circ} \mathrm{C} / \mathrm{s}$; internal pressure of 7 bar is imposed at $t=30 \mathrm{~s}\left(T=40^{\circ} \mathrm{C}\right)$; elongation in the $\theta$-direction stops at $t \cong 90 \mathrm{~s}\left(T \cong 70^{\circ} \mathrm{C}\right)$ when the sample meets the mold walls. After $t \cong 110 \mathrm{~s}$, the sample is isothermal at $80^{\circ} \mathrm{C}$. 